\title{
Study the Small and Dense Lipoprotein Levels (LDLsd) in Coronary Artery Disease
}

\author{
Agustin Nestor Joison $^{1^{\star}}$, Sergio Rufino ${ }^{1}$ and Enrique Majul ${ }^{2}$ \\ ${ }^{1}$ Associate Professor of Medical Chemistry III, Faculty of Medicine, UCC, Argentina \\ ${ }^{2}$ Chairman of Medical Chemistry III, Faculty of Medicine, UCC, Argentina
}

"Corresponding author: Agustin Nestor Joison, Associate Professor of Medical Chemistry III, Faculty of Medicine, UCC, Dean Funes 1752 Tower I Floor 9 dep C, 5003 Córdoba, Argentina, Tel: +5493518407421; Fax: +5493514807421; E-mail: ajoison2001@yahoo.com.ar

Rec Date: Feb 3, 2014, Acc Date: Apr 16, 2014, Pub Date: Apr 22, 2014

Copyright: (C) 2014 Joison AN, et al. This is an open-access article distributed under the terms of the Creative Commons Attribution License, which permits unrestricted use, distribution, and reproduction in any medium, provided the original author and source are credited.

\begin{abstract}
Objective: To study the height LDLsd levels and coronary disease relation.

Methods: Experimental transversal and retrospective study of sample not probabilistic random. 62 individuals were studied of both sex who did attended a consultation specialized in cardiology at the University Queen Fabiola Clinic of Cordoba city Argentina. The LDLsd are separated using vertical polyacrylamide gradient gel electrophoresis to $3 \%$ in non-reducing conditions. The presence of LDLsd was established when identified in the serum LDLsd abnormal band with $\mathrm{Rf}>0.36$.
\end{abstract}

Results: 26 patients have LDLsd elevated and 36 patients with normal LDLsd $\mathrm{Rf}<0.36)$. In $36(58 \%)$ patients with normal LDLsd, $27(75 \%)$ of them have not coronary disease and diabetes. A correlation study between factors of risk and coronary disease with a $95 \%$ of statically significance resulted that 16 patients with coronary disease had height LDLsd $(\mathrm{Rf}>0.36)$ and 7 patients without disease $(p<0.001)$.

Conclusion: The research revealed high levels of LDLsd in subjects assessed, by association with coronary disease increased significantly. Patients with normal TGR who had elevated levels of LDLsd had developed coronary artery disease; it confirms to LDLsd as independent of coronary disease risk factor.

Keywords: LDLsd; Coronary disease; Triglycerides; Hypertension; Diabetics

\section{Introduction}

Cardiovascular disease as atherosclerosis result, continue being the first cause of death in develop countries and growing in the others culture. In the last years important advances have been made in the knowledge of the pathogenesis, course and treatment of this disease. It has been established at molecular and cellular levels the transcendental role of lipid metabolism disorders in the development of atherosclerotic pathology. This knowledge has led if the dyslipoproteinemia treatment will could make reduced to cardiovascular diseases in the future [1].

During the early stage in the development of atherosclerosis, low density lipoproteins (LDL) which have the function of cholesterol transport in blood, are mainly involved in that process. On the arterial surface these particles of LDL are hydrolyzed and as part of that biochemical modification, some may penetrate through the intimal, where by oxidative processes of the lipoprotein lipids, are modified so that they are incorporated by macrophages and as result in foam cells formation $[2,3]$.

Not always increases in apo B have to be accompanied by elevation of cholesterol since this can be normal. Alternatively there may be a hiperapobetalipoproteinemia which is characterized by formation of LDL more small and dense with greater power atherogenic. It is known that there are pathologies such as insulin resistance where can be associated with the increase of the LDLsd and an increase of triglyceride in blood $[4,5]$. The LDLsd contains less cholesterol but higher of apo B levels, making them much more for oxidation and therefore more atherogenic with respect to other subclasses of LDL particles [6]. Another important fact is that the LDLsd increased in insulin resistance disease is often associated with the women menopausal $[7,8]$.

It is known that the cholesterol have not only the ability to pass through the vascular endothelium into the intimate. The metabolism provides lipoproteins for its transport and the LDLsd due to their size and density can through it below vascular sub endothelium. There is a relationship between the size of lipoprotein and endothelial damage while small is the LDLsd bigger is the atherogenic effect. The decrease in the size and density of the LDL does have up to $40 \%$ more than odds infiltrate to the intimate and increase its atherogenic effect [9-12].

Relating the atherogenic damage with coronary disease process, many studies made it possible to demonstrate that the size and density of the LDLsd could have a predictor factor of the disease. These factors are due to phenotypic determinant of LDL and therefore it is very important for the pharmacological treatment the pathology, modifying the susceptibility to oxidation of the particle as well as his movement into the intimate [13].

A variation of $30 \%$ to $60 \%$ in the size of LDL particles is explained by genetic factors Genetic factors with information including the Apo E, hepatic lipase, cholesterol (CETP) ester transfer protein, lipoprotein 
lipase (LPL) and the cluster of ApoA1/C3/A4/A5. But we must consider to habits such as food, sedentary lifestyle, diseases as obesity, insulin resistance, diabetes, etc. also has an effect on the size of the LDL lipoproteins [14].

\section{Methods}

Experimental transversal and prospective study of sample not probabilistic and random 62 individuals were studied of both sexes who did attend a consultation specialized in cardiology at the University Queen Fabiola Clinic of Cordoba city Argentina. The study was carried out from July 2010 to July 2012, which met the following inclusion criteria: age $\geq 18$ years with personal medical history of cardiovascular disease (acute infarction myocardium, ischemic heart disease, heart failure, stroke, peripheral arterial disease and deep venous thrombosis), renal disease, diabetes mellitus or other endocrine pathology, liver disease and cancer. The patients were not treated with statins or drugs that will be modified the lipids or lipoproteins levels.

Pregnant women were excluded. All the agreements of the Declaration of Helsinki, obtaining signed informed consent of individuals studied were approved. By clinical history were obtained personal data and demographic, personal and family history in the first degree of consanguinity of arterial hypertension (AHT), ischemic heart disease (ECI), stroke (stroke), diabetes mellitus (DM) and other personal medical history, smoking, consumption of alcohol and drug treatment.

For the study of the LDLsd, $10 \mathrm{~mL}$ of blood was extracted by venous puncture in the crease of the elbow, prior fasting for 12-14 hours. After centrifugation, the extracted serum was distributed in three aliquots, using the first for the determination of indicators of lipid profile; the remaining stored in tubes eppendorf at $-20^{\circ} \mathrm{C}$ until the moment in which analyses were carried out. The day of the test sample was determined in serum levels of small, dense LDL. The LDLsd are separated using vertical polyacrylamide gradient $3 \%$ gel electrophoresis in non-reducing conditions. $50 \mathrm{ul}$ of total serum is mixed with $50 \mathrm{ul}$ of sudan black at $0.1 \%$ and planted in the gel, $110 \mathrm{~V}$ for 35 minutes in Tris buffer ( $\mathrm{pH} \mathrm{8.3)} \mathrm{glycine} \mathrm{at} 4^{\circ} \mathrm{C}$.

Characterization of the LDLsd bands was carried out based on Rf (relative mobility). Each LDLsd band Rf is calculated follows: the presence of LDLsd was established when identified in the serum LDLsd abnormal band with Rf $>0.36$.

The statistical analysis was conducted with the SPAD (V.5.6) software. The value of $p$ less than 0.05 was considered significant. The data obtained were analyzed by multivariate analysis.

\section{Results}

After analysis of 62 patients there are 26 patients with LDLsd elevated ( $\mathrm{Rf}>0.36$ ) and 36 patients with normal LDLsd $\mathrm{Rf}<0.36$ ). According to the analysis in 36 (58\%) patients with normal LDLsd, 27 $(75 \%)$ of them have not coronary disease and diabetes, 5 (13.8\%) have coronary disease, $2(5.5 \%)$ are diabetics and $2(5.5 \%)$ have coronary disease and diabetes (Figure 1A). In 26 (42\%) patients with elevated LDLsd, 7 (26.9\%) have not coronary disease and diabetes, 13 (50\%) have coronary disease $3(11.5 \%)$ are diabetics and $3(11.5 \%)$ have both pathologies (Figure 1B). Respect to 57 (91.9\%) patients with triglycerides elevated (> $170 \mathrm{mg} \%), 33(5.8 \%)$ have not coronary disease and diabetes, $14(24,6 \%)$ have coronary disease, $7(12.2 \%)$ are diabetics and 3 (5.2\%) have coronary disease and diabetes (Figure 2A). 5 patients (8\%) with normal triglycerides, 2 (40\%) have not coronary disease and diabetes, $2(40 \%)$ have coronary disease and $1(20 \%)$ have coronary disease and diabetes. There are not patients with diabetes in this group (Figure 2B). When study 9 (14.5\%) patients with hypertension (>90/150 $\mathrm{mmHg}), 2(22.2 \%)$ have not coronary disease and diabetes, 4 (44.4\%) have coronary disease, 1 (11.1\%) are diabetics, and $2(22.2 \%)$ have coronary disease and diabetes (Figure 3A). 53 patients $(85.4 \%)$ with hypertension, $33(62.2 \%)$ have not coronary disease and diabetes, $12(23.6 \%)$ have coronary disease, $2(3.7 \%)$ are diabetics and $6(11.3 \%)$ have coronary disease and are diabetics (Figure 3B). A correlation study between factors of risk and coronary disease with a $95 \%$ of statically significance resulted that 16 patients with coronary disease had height LDLsd $(\mathrm{Rf}>0.36)$ and 7 patients without disease $(\mathrm{p}<0.001)$ (Table 1$)$.

\begin{tabular}{|l|l|l|l|}
\hline & coronary disease & No coronary disease & $\mathrm{p}$ \\
\hline LDLsd elevated & $16(61.5 \%)$ & $7(26.9 \%)$ & $<0.002$ \\
\hline TGR elevados & $14(24.5 \%)$ & $33(57.8 \%)$ & $<0.5$ \\
\hline Hypertensos & $4(44.4 \%)$ & $2(22.2 \%)$ & $<0.18$ \\
\hline
\end{tabular}

Table 1: Table showing the statist significance with different risk factors

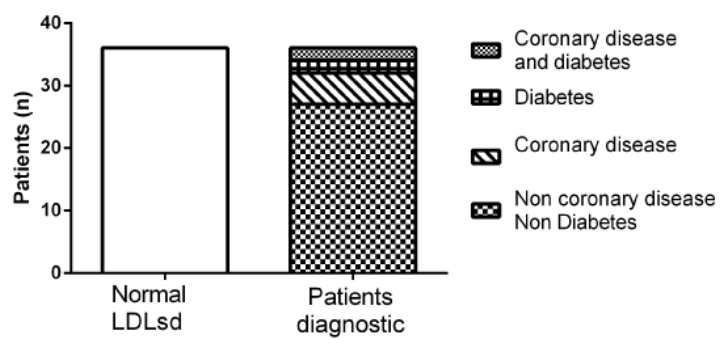

Fig $1 \mathrm{~A}$

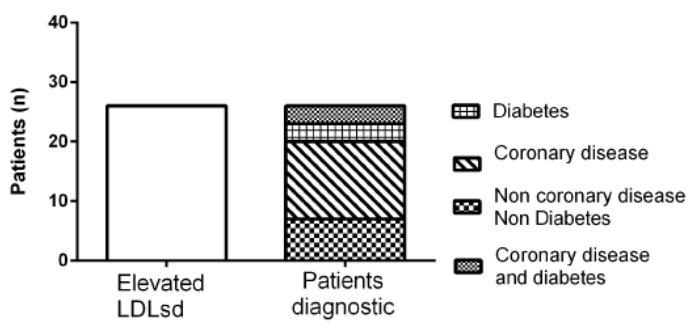

Fig 1 B

Figure 1A: LDLsd normal in coronary disease and diabetes, Figure 1B: LDLsd elevated in coronary disease and diabetes 
Page 3 of 4

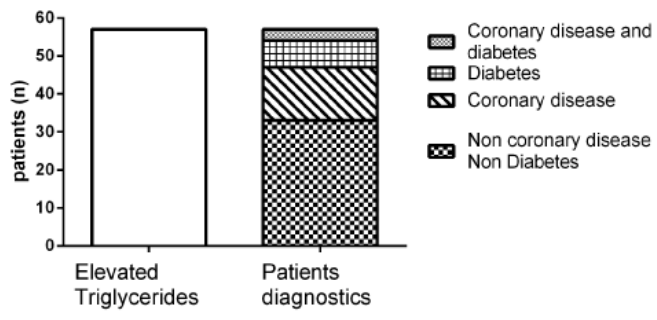

Fig 2 A

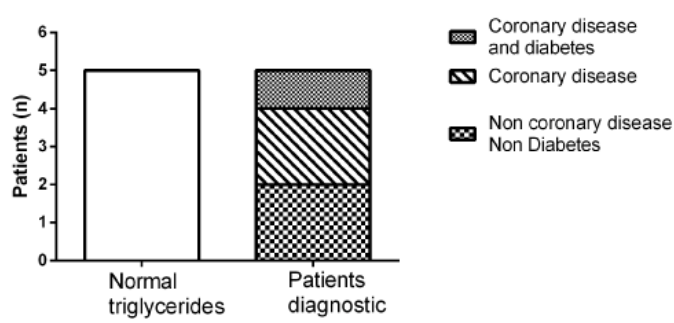

Fig 2 B

Figure 2A: Triglycerides elevated in coronary disease and diabetes, Figure 2B: Triglycerides normal in coronary disease and diabetes

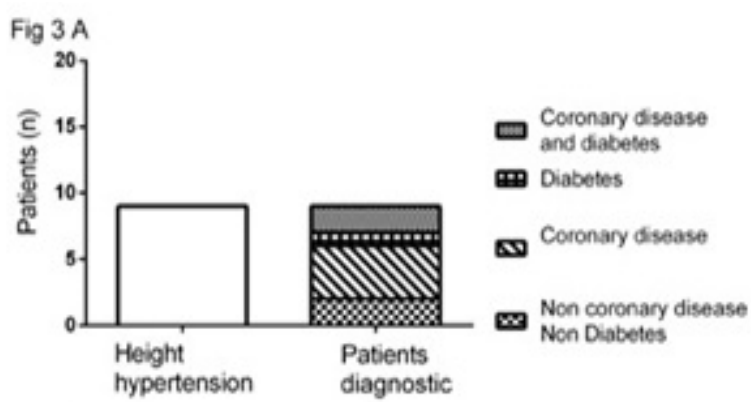

Fig 3 B

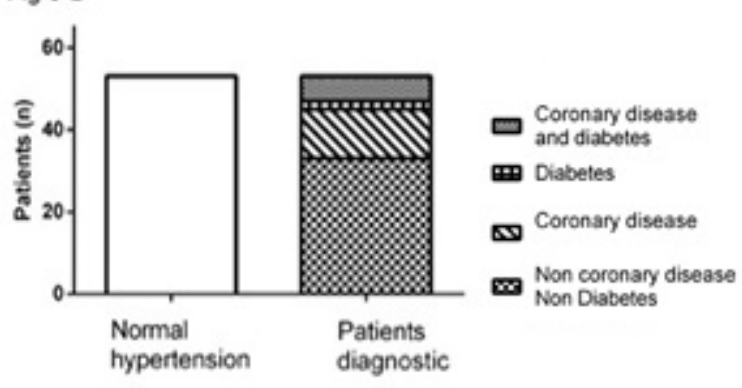

Figure 3A: Height hypertension in coronary disease and diabetes, Figure 3B: Normal hypertension in coronary disease and diabetes

\section{Discussion}

Historically has been considered the increase of the concentration of LDL in blood as a cardiovascular risk factor, the size and density of the LDL emerges as a new and important risk factor for these diseases. Although the mechanism has not been well elucidated, there is evidence that LDLsd is more susceptible to oxidation and increase the risk of cardiovascular disease. The oxidized LDL itself then becomes more reactive with the surrounding tissues, which can produce tissue damage [15]. The present study allowed us to observe that many patients with normal LDL in blood, their LDLsd is increased. Regards to cardiovascular risk, the characteristics of size and density of the LDL are as important as its concentration in blood.

In 26 patients (41.9\%) of the sample was detected LDLsd increased, a higher percentage than expected, because literature has pointed out that the prevalence of this type of LDL particle is $30-35 \%$. It has been suggested that LDL size is inversely proportional to dietary intake of carbohydrates as the prevalence of apo B (apoprotein B) from LDL phenotype, increasing directly with pathologies such as diabetes [16]. The results show it is possible that the high prevalence of LDLsd in the sample studied is related with this disease, because $10 \%$ of all diabetic patients have elevated the LDLsd.

Although the complexity of the method for determining the levels of LDLsd could be a limitation, it is important to do because it regards this lipoprotein as cardiovascular risk factors such concentration of triglycerides, cholesterol high-density (HDL-C) lipoprotein and apolipoprotein B. As noted, the highest proportion of LDLsd is observed when we have high triglyceride concentration $>134 \mathrm{mg} / \mathrm{dl}$ and low $(<40)$ HDL cholesterol concentration, which allows estimating the presence of LDLsd by the TG/HDL-C ratio, with a cutoff point of 1.35. Contrary to this in our study, we detected that 25 patients with high LDLsd, $22 \%$ of them have normal triglycerides. Abnormalities of LDL sub-fractions are an aspect to consider between hypertensive patients, since these individuals already possess a high cardiovascular risk.

There is evidence of the association between high blood pressure and LDLsd where other authors have reported, even without evidence of an altered lipid profile. Increased frequency of observed LDLsd among hypertensive probably has its origin in the close relationship that keeps hypertension and abdominal obesity [17]. According to the analysis made in our study (14.5\%) patients with hypertension, 2 $(22.2 \%)$ have not coronary disease and diabetes, 4 (44.4\%) have coronary disease, $1(11.1 \%)$. And $5 \%$ patients with hypertension have coronary disease and LDLsd elevated.

One of the difficulties in trying to determine whether the LDLsd is an independent predictor of the risk of coronary heart disease or merely a marker of other lipid abnormalities is that the LDLsd is associated with concentrations of triglycerides, reduced cholesterol HDL, and insulin resistance. Lipid profile characteristic of metabolic syndrome (SM), diabetes mellitus, hypertriglyceridemia from any cause and chronic renal failure, among others [18]. Our results in this sample of patients, allowed us to observe that patients with elevated triglycerides 14 patients have coronary heart disease. This could explain the LDLsd to behave like an independent risk factor.

In addition, found an association between the frequency of LDLsd and smoking. These results agree with those obtained by to the. exposure to nicotine reduces the release of insulin and adversely affects the action of this hormone, so it is likely that the insulin resistance is the link between the effects of smoking and this type of 
particles of LDL, observed among smokers in addition to higher levels of cholesterol and triglycerides, lower sensitivity to insulin and increased activity of hepatic lipase an enzyme which as previously noted is involved in the formation of LDLsp. The results of this study showed that of those patients with LDLsd elevated are smokers and have coronary disease, confirming the importance of the relationship between smoking, LDLsd and coronary heart disease.

\section{Conclusion}

In conclusion, the research revealed high levels of LDLsd in subjects assessed, by association with coronary disease increased significantly. Patients with normal TGR who had elevated levels of LDLsd developed coronary artery disease; it confirms to LDLsd as independent of coronary disease risk factor.

\section{Acknowledgement}

To the coronary unit of Reina Fabiola Clinic, Christine Stevenazzi biochemistry chair of central laboratory

\section{References}

1. Brown MS, Goldstein JL (1996) Heart attacks: gone with the century? Science 272: 629.

2. Witztum JL, Steinberg D (1991) Role of oxidized low density lipoprotein in atherogenesis. J Clin Invest 88: 1785-1792.

3. Esterbauer H, Gebicki J, Puhl H, Jürgens G (1992) The role of lipid peroxidation and antioxidants in oxidative modification of LDL. Free Radic Biol Med 13: 341-390.

4. Austin MA, King MC, Vranizan KM, Krauss RM (1990) Atherogenic lipoprotein phenotype. A proposed genetic marker for coronary heart disease risk. Circulation 82: 495-506.

5. Austin MA, Edwards KL (1996) Small, dense low density lipoproteins, the insulin resistance syndrome and noninsulin-dependent diabetes. Curr Opin Lipidol 7: 167-171.

6. Berneis KK, Krauss RM (2002) Metabolic origins and clinical significance of LDL heterogeneity. J Lipid Res 43: 1363-1379.
7. Ayyobi AF, Brunzell JD (2003) Lipoprotein distribution in the metabolic syndrome, type 2 diabetes mellitus, and familial combined hyperlipidemia. Am J Cardiol 92: 27J-33J.

8. Berg G, Muzzio ML, Wikinski R, Schreier L (2004) A new approach to the quantitative measurement of dense LDL subfractions. Nutr Metab Cardiovasc Dis 14: 73-80.

9. Rizzo M, Berneis K, Corrado E, Novo S (2006) The significance of lowdensity-lipoproteins size in vascular diseases. Int Angiol 25: 4-9.

10. St-Pierre AC, Cantin B, Dagenais GR, Després JP, Lamarche B (2006) Apolipoprotein-B, low-density lipoprotein cholesterol, and the long-term risk of coronary heart disease in men. Am J Cardiol 97: 997-1001.

11. Todd J, Farmer JA (2006) Optimal low-density lipoprotein levels: evidence from epidemiology and clinical trials. Curr Atheroscler Rep 8: 157-162.

12. Rizzo M, Berneis K (2006) Low-density lipoprotein size and cardiovascular prevention. Eur J Intern Med 17: 77-80.

13. Hirany SV, Othman Y, Kutscher P, Rainwater DL, Jialal I, et al. (2003) Comparison of low-density lipoprotein size by polyacrylamide tube gel electrophoresis and polyacrylamide gradient gel electrophoresis. Am J Clin Pathol 119: 439-445.

14. Ramakrishnan L, Prabhakaran M, Ruby G, Kolli Srinath R (2012) LDL particle heterogeneity and its association with other established cardiovascular risk factors in a young Indian industrial population. Heart Asia 4: 141-145.

15. Otvos JD, Jeyarajah EJ, Cromwell WC (2002) Measurement issues related to lipoprotein heterogeneity. Am J Cardiol 90: 22-29.

16. Scheffer PG, Teerlink T, Heine RJ (2005) Clinical significance of the physicochemical properties of LDL in type 2 diabetes. Diabetologia 48 : 808-816.

17. Mathieu P, Poirier P, Pibarot P, Lemieux I, Després JP (2009) Visceral obesity: the link among inflammation, hypertension, and cardiovascular disease. Hypertension 53: 577-584.

18. Silliman K, Shore V, Forte TM (1994) Hypertriglyceridemia during late pregnancy is associated with the formation of small dense low-density lipoproteins and the presence of large buoyant high-density lipoproteins. Metabolism 43: 1035-1041. 